\title{
Splint maxilar modificado como auxiliar na correção da má oclusão de Classe II, Divisão 2, sem crescimento: relato clínico
}

\author{
Daltro RITTER*, Arno LOCKS**, Luiz G. GANDINI JR.***, Renésio A. GREHS*, Maurício Tatsuei SAKIMA***
}

\begin{abstract}
Resumo
Neste artigo é descrito o caso clínico de um paciente adulto, sem crescimento, com má oclusão de Classe II, divisão 2, tratado com um splint maxilar modificado. Foi exercida força extrabucal com direção de tração parietal com força de 400 gramas, com uso diário de 14 horas durante 1 ano. Com este aparelho removivel corrigiu-se completamente a relação de Classe II dos molares e pré-molares, levando estes dentes à oclusão normal, não havendo extrusão dentária, mantendo constante o plano mandibular. Após a correção dos dentes posteriores com o splint maxilar modificado, utilizou-se aparelhagem fixa convencional para corrigir as inclinações dos dentes anteriores e finalizar o caso.
\end{abstract}

Palavras-chave: Classe II, divisão 2. Splint maxilar. Adulto.

\section{INTRODUÇÃO}

Atualmente tem-se verificado um número crescente de pacientes que procuram tratamento ortodôntico na idade adulta. Estes são caracterizados por terem ausência de crescimento e, portanto, quando uma má oclusão está instalada o tratamento disponível é a movimentação dentária, sem a possibilidade de guiar ortopedicamente o crescimento dos maxilares. Em situações em que existe uma relação esquelética muito desfavorável entre a maxila e a mandíbula, a Ortodontia aliada à Cirurgia Ortognática pode ser uma opção de tratamento.

Segundo Thurow ${ }^{13}$, nos pacientes em crescimento, o aparelho extrabucal com splint maxilar é um método efetivo para a correção ortopédica inicial da Classe II com componentes esqueléticos. Outros autores recomendam esse tipo de aparelho para melhorar a relação maxilo-mandibular e o posicionamento dentário, facilitando o tratamento ortodôntico posterior ${ }^{1,6,7}$. Devido à possibilidade de incorporar neste aparelho todos os dentes irrompidos, decíduos ou permanentes, existe maior área de atuação das forças quando comparado a aparelhos que utilizam somente os primeiros molares permanentes superiores como ancoragem $^{5,8,12}$.

Existem muitos trabalhos na literatura mostrando os efeitos do splint maxilar de Thurow em pacientes com má oclusão de Classe II, divisão 1,

Aluno do Curso de Doutorado em Ortodontia e Ortopedia Facial da UNESP-Araraquara-SP.

** Professor Doutor Coordenador do Curso de Especialização em Ortodontia e Ortopedia Facial da UFSC - Florianópolis-SC

*** Professor Doutor do Departamento de Clínica Infantil e do Curso de Doutorado em Ortodontia e Ortopedia Facial da UNESP - Araraquara - SP. 
com crescimento disponível, mas não há relatos do uso deste aparelho em pacientes com má oclusão de Classe II, divisão 2, sem crescimento 1,2,3,6,10,11,14. Este artigo objetiva evidenciar as alterações dentárias e esqueléticas ocorridas no tratamento de um paciente portador de má oclusão de Classe II, divisão 2, de Angle, sem crescimento, através do uso de um splint maxilar modificado.

\section{CASO CLÍNICO}

Paciente do gênero masculino, 18 anos de idade, perfil levemente convexo (Fig. 1), no estágio de dentadura permanente com os molares em relação de Classe II completa e os incisivos superiores retroinclinados, caraterizando uma má oclusão Classe II, divisão 2 de Angle (Fig. 2). O arco inferior apresentava-se bem alinhado e nivelado, com os incisivos inferiores bem posicionados. Cefalometricamente verificou-se um ANB de 6 graus, ângulo de convexidade de 9 graus, plano mandibular de 25 graus e FMA de 20 graus. Os incisivos superiores apresentavam-se extremamente retroinclinados, com 1.NB de 1 grau, com o ângulo interincisal de 151 graus (Tab. 1).

Apesar do paciente não apresentar crescimento, decidiu-se utilizar inicialmente o splint maxilar para corrigir o relacionamento dentário posterior por distalização dos pré-molares e molares superiores. Devido à inclinação vertical dos incisivos superiores e conseqüente ausência de overjet, não se incorporou ao aparelho os incisivos e os caninos, englobando em sua estrutura somente as coroas dos molares e pré-molares superiores (Fig. 3). Foi exercida força extrabucal de puxada alta com aproximadamente 400 gramas bilateralmente, sendo prescrito ao paciente o uso do splint maxilar 14 horas por dia. O arco externo do aparelho foi encurtado e inclinado superiormente, para melhor distribuir a força e evitar inclinações dentárias.

Após 1 ano de uso do splint maxilar, obtevese o relacionamento de molares e pré-molares em Classe I, não alterando a relação entre os incisivos superiores e inferiores, havendo abertura de espaço na distal dos caninos superiores (Fig. 4). Nesta fase de reestudo os incisivos superiores e inferiores apresentaram poucas alterações clínicas e cefalométricas (Tab. 1). Após corrigida a relação dentária posterior, foi instalado aparelho fixo em ambas os arcos, realizadas as fases de alinhamento e nivelamento com fios redondos, e distalização dos caninos superiores até atingir relação de Classe I. Durante o período de tratamento com os aparelhos fixos,
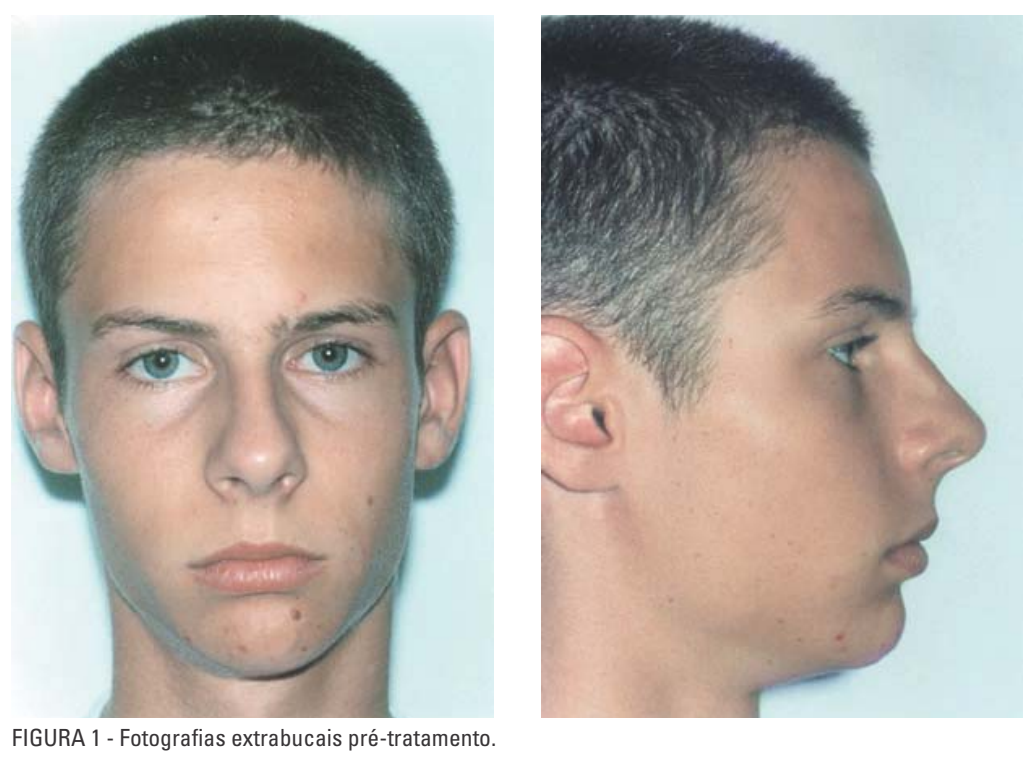

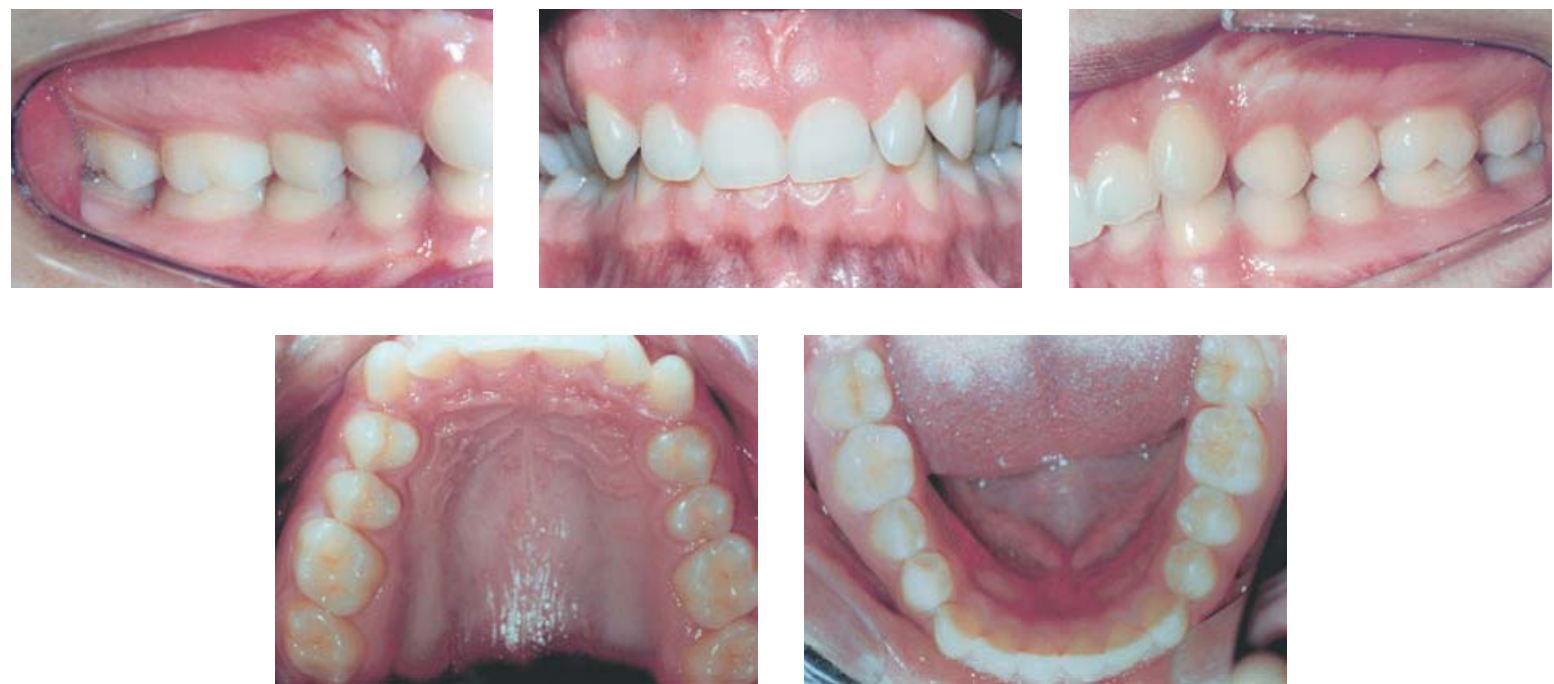

FIGURA 2 - Fotografias intrabucais pré-tratamento. Notar a relação de molares e caninos em Classe II, e os incisivos centrais superiores retro-inclinados.
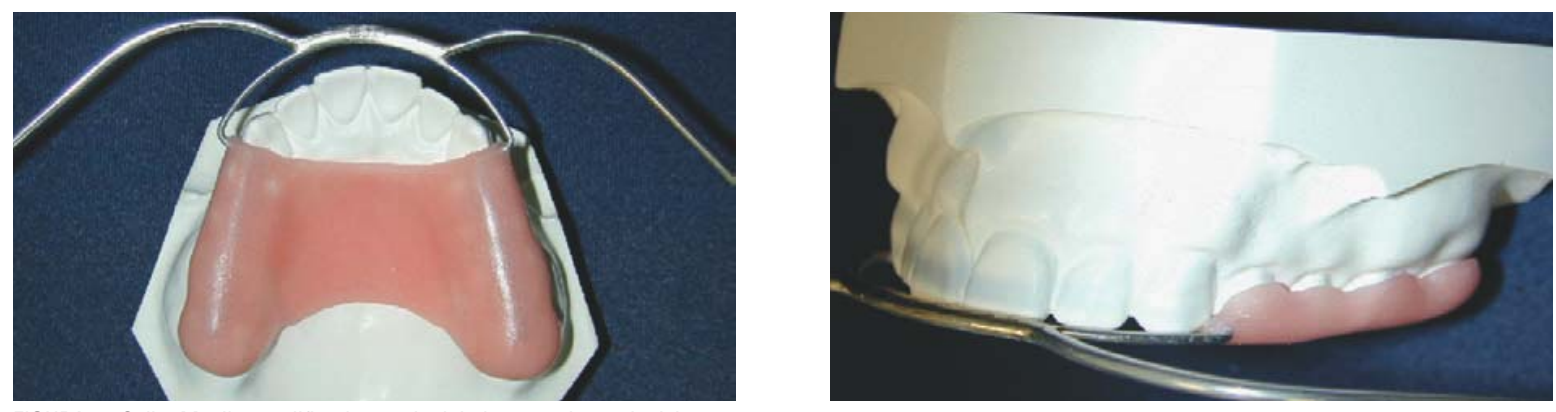

FIGURA 3 - Splint Maxilar modificado, não incluindo os caninos e incisivos.
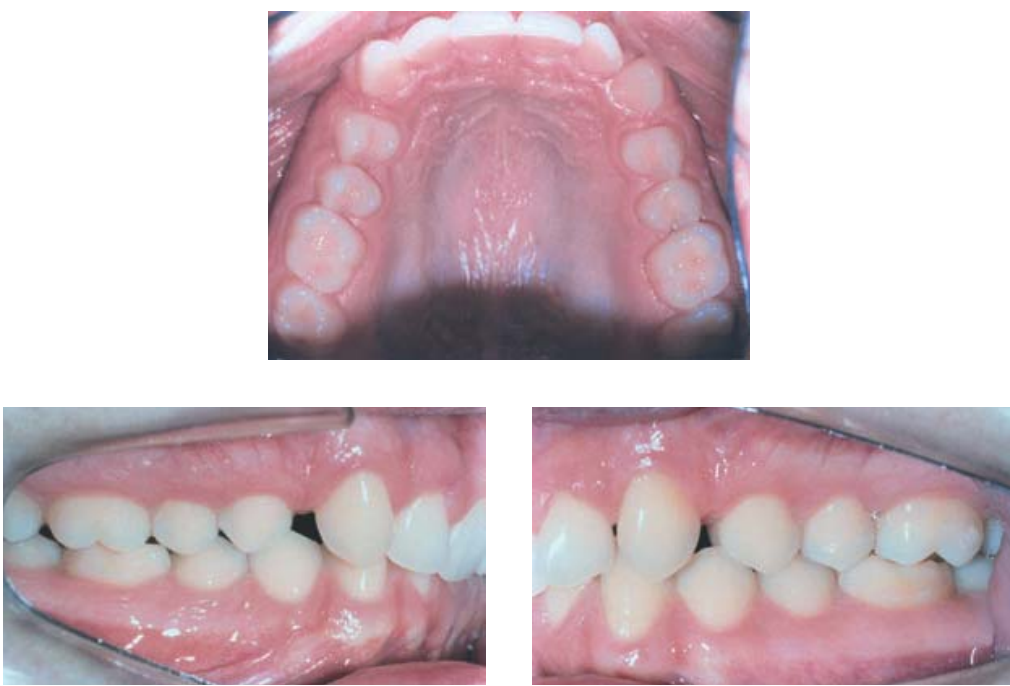

FIGURA 4 - Fotografias intrabucais pós-utilização do splint maxilar. Notar a relação de molares e pré-molares em Classe I, e o espaço existente na distal dos caninos superiores. 

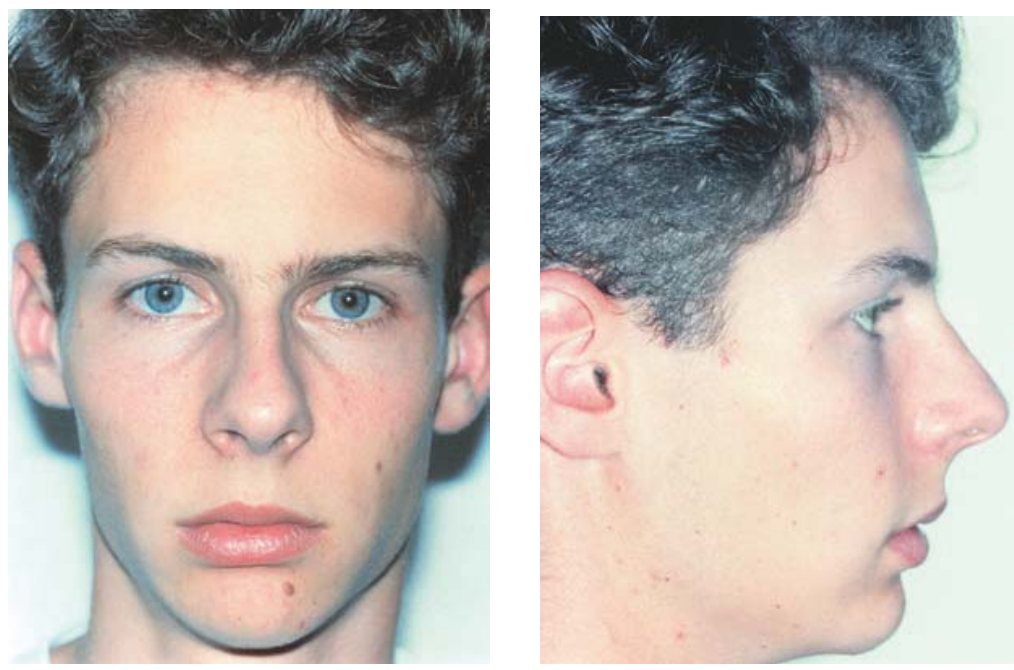

FIGURA 5 - Fotografias extrabucais finais.
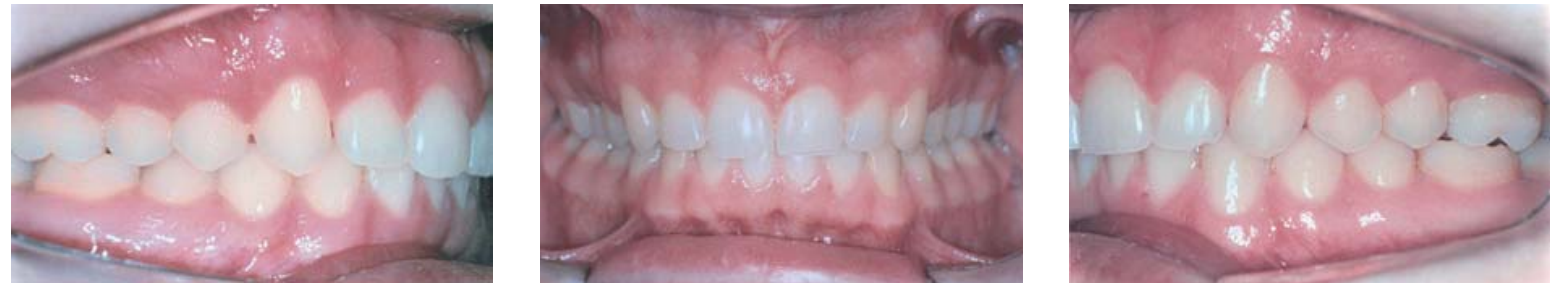

FIGURA 6 - Fotografias intrabucais finais. Notar a relação de molares e caninos em Classe I, e a correção da posição dos incisivos superiores.

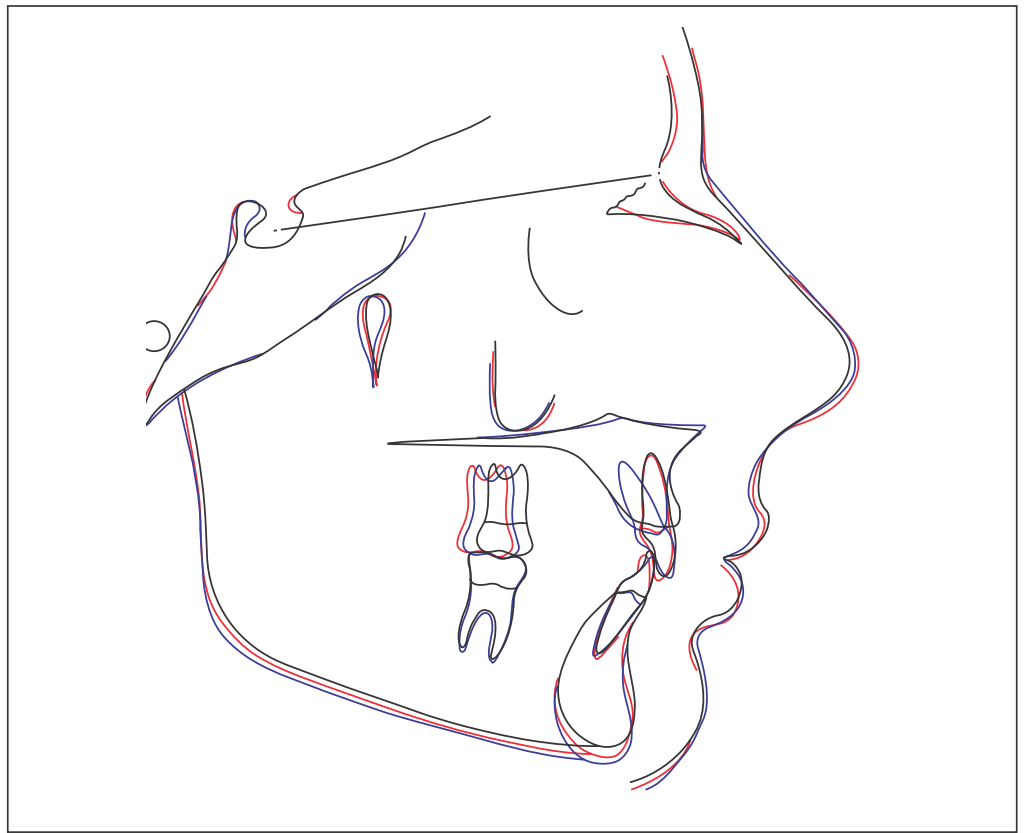

FIGURA 7 - Superposição total em SN dos traçados cefalométricos nos períodos inicial (preto), reestudo (vermelho) e final (azul). 


\begin{tabular}{|c|c|c|c|}
\hline \multicolumn{3}{|c|}{ Tabela 1 - Medidas cefalométricas iniciais, de reestudo e } \\
\hline finais. \\
\hline Medida & Inicial & Reestudo & Final \\
\hline SNA & $83^{\circ}$ & $82^{\circ}$ & $82^{\circ}$ \\
\hline ANB & $77^{\circ}$ & $76^{\circ}$ & $76^{\circ}$ \\
\hline SND & $6^{\circ}$ & $6^{\circ}$ & $6^{\circ}$ \\
\hline NAP & $75^{\circ}$ & $75^{\circ}$ & $74^{\circ}$ \\
\hline Ângulo facial & $10^{\circ}$ & $10^{\circ}$ & $6^{\circ}$ \\
\hline Eixo Y & $87^{\circ}$ & $86^{\circ}$ & $85^{\circ}$ \\
\hline GoGn.SN & $58^{\circ}$ & $59^{\circ}$ & $60^{\circ}$ \\
\hline FMA & $25^{\circ}$ & $25^{\circ}$ & $25^{\circ}$ \\
\hline FMIA & $20^{\circ}$ & $20^{\circ}$ & $19^{\circ}$ \\
\hline IMPA & $62^{\circ}$ & $63^{\circ}$ & $60^{\circ}$ \\
\hline $1 . \mathrm{Na}$ & $98^{\circ}$ & $97^{\circ}$ & $101^{\circ}$ \\
\hline 1-Na & $1^{\circ}$ & $1^{\circ}$ & $22^{\circ}$ \\
\hline $1 . \mathrm{Nb}$ & $2 \mathrm{~mm}$ & $0,5 \mathrm{~mm}$ & $3 \mathrm{~mm}$ \\
\hline 1-Nb & $23^{\circ}$ & $20^{\circ}$ & $24^{\circ}$ \\
\hline 1.1 & $4 \mathrm{~mm}$ & $4 \mathrm{~mm}$ & $5 \mathrm{~mm}$ \\
\hline Plano oclusal & $151^{\circ}$ & $152^{\circ}$ & $130^{\circ}$ \\
\hline Pog-NB & $15^{\circ}$ & $15^{\circ}$ & $15^{\circ}$ \\
\hline Ls-S & $2,5 \mathrm{~mm}$ & $3 \mathrm{~mm}$ & $3 \mathrm{~mm}$ \\
\hline Li-S & $-0,5 \mathrm{~mm}$ & $-1,5 \mathrm{~mm}$ & $-2 \mathrm{~mm}$ \\
\hline & $-1 \mathrm{~mm}$ & $-1 \mathrm{~mm}$ & $0 \mathrm{~mm}$ \\
\hline
\end{tabular}

que durou 14 meses, o paciente utilizou arco extrabucal noturno, com tração alta, força bilateral de 400 gramas, com objetivo de manter as posições dentárias dos molares e pré-molares. Ao final de todo o tratamento, a face do paciente sofreu poucas alterações (Fig. 5), verificou-se manutenção na medida ANB e do ângulo de convexidade. O plano mandibular e o FMA mantiveram-se constantes durante o tratamento, assim como o plano oclusal (Tab. 1). A relação inter-incisal foi corrigida com arcos retangulares, proporcionando torque radicular palatino adequado a estes dentes (Fig. 6).

\section{DISCUSSÃO}

Pacientes sem crescimento, com relação incorreta entre a maxila e a mandíbula no sentido ântero-posterior, como na Classe II, tem como uma alternativa de tratamento a distalização dos dentes superiores.
Isto requer colaboração por parte do paciente, o que irá influenciar no tempo e no resultado final do tratamento. No caso clínico descrito neste artigo, houve uma excelente colaboração, o que permitiu que um resultado favorável fosse obtido. Nos 12 primeiros meses de tratamento o único dispositivo utilizado foi o splint maxilar removível, sendo que aparelhos fixos foram instalados nos 14 meses finais. Isto pode ser considerado uma vantagem, visto diminuir o tempo total do tratamento com dispositivos fixos cimentados, reduzindo o risco de cáries, descalcificações, traumas com o aparelho, quebras destes dispositivos, podendo os mesmos serem considerados esteticamente desagradáveis pelos pacientes.

Com mais dentes incorporados ao aparelho, o splint maxilar permite aumentar o limite de força à disposição para tração extrabucal ${ }^{8}$. Embora alguns autores empreguem forças mais altas e por mais horas diariamente $\mathrm{e}^{1,3,6,12}$, a utilização do aparelho com 400 gramas de força em torno de 14 horas diárias, como o fez Thurow ${ }^{13}$ e outros ${ }^{4,8,9}$, mostrou-se eficaz, corrigindo a má oclusão de Classe II exclusivamente através de movimentação dentária.

No caso clínico deste estudo, os incisivos superiores e inferiores sofreram poucas alterações nas suas posições durante a primeira fase do tratamento com o splint maxilar, pois não estavam incorporados ao aparelho. Nesta primeira fase, houve distalização dos molares e pré-molares, corrigindo a relação destes dentes até atingir Classe I. Os ângulos SNA, SNB e convexidade facial permaneceram inalterados, indicando não haver influência direta do aparelho sobre as bases ósseas no sentido horizontal. Houve controle sobre a extrusão dentária, evitando a abertura do plano mandibular (GoGn.SN) no sentido vertical. Quando ocorre giro mandibular no sentido horário, o ponto $\mathrm{B}$ passa a localizar-se mais posteriormente, aumentando a discrepância horizontal entre as bases ósseas ${ }^{4,14}$. Desta maneira, o controle sobre os componentes verticais de extrusão dentária aliado à correção horizontal dentária, atingiram os objetivos propostos de evitar a abertura do plano mandibular 
e correção dentária da má oclusão, facilitando a finalização posterior com aparelhagem fixa.

Verificou-se assim a possibilidade de utilizar o splint maxilar modificado com objetivo de movi- mentação dentária em bloco. Como vantagens verificou-se a possibilidade de distalizar dentes evitando sua extrusão, controlar o plano mandibular e protelar a instalação do aparelho fixo.

\title{
Modified maxillary splint as an auxiliary in without growth Class II Division 2 malocclusion correction: Clinical Report
}

\begin{abstract}
In this article is reported a Class II division 2 clinical case of an adult without growth treated with a modified maxillary splint. It was used extra-buccal force with parietal traction with 400 grams of force 14 hours a day. With this removable device it was corrected the molars and bicuspids Class II relation without extrusion and then controlling the opening of the mandibular plane. After the correction of the posterior teeth with the modified maxillary splint, it was utilized conventional fixed orthodontic therapy to correct the inclinations of the anterior teeth and finish the case.
\end{abstract}

Key words: Class II division 2. Maxillary splint. Adult.

\section{REFERÊNCIAS}

1. BASS, N. M. Orthopedic coordination of dentofacial development in skeletal Class II malocclusion in conjunction with Edgewise therapy. Part I. Am J Orthod, St. Louis, v. 84, no. 5, p. 361-383, 1983.

2. BAUMRIND S.; KORN, E. L.: ISAACSON, R. J.; WEST, E. E. MOLTEN, R. Quantitative analysis of the orthodontic and orthopedic effects of maxillary traction. Am J Orthod, St. Louis, v. 84 no. 5, p. 384-398, 1983.

3. CALDWELL, S. F.; HYMAS, T. A.; TIMM. T. A. Maxillary traction splint: a cefalometric evaluation. Am J Orthod, St. Louis, v. 69 no. 5, p. 376-384, 1984

4. FOTIS, V.; MELSEN, B.; WILLIAMS, S. Vertical control as an important ingredient in the treatment of severe sagittal discrepancies. Am J Orthod, St. Louis, v. 86, no. 3, p. 224-232, 1984

5. HENRIQUES, J. F. C.; RODRIGUES, D. Modified maxillary splint for Class II. Division 1 Treatment. J Clin Orthod, Boulder, v. 25 no. 4, p. 239-245, 1991

6. JOFFE, L.; JACOBSON, A. The maxillary orthopedic splint. Am J Orthod, St. Louis, v. 75, no. 1, p. 54-69, 1979

7. KING, G. J.; KEELING, S. D.; HOCEVAR, R. A.; WHEELER, T. T. The timing of treatment for Class II malocclusions in children: a literature review. Angle Orthod, Appleton, v. 60, no. 2, p. 87-97, 1990.

8. MENEZES, L. M.; LIMA, E. M. S.; BOLOGNESE, A. M. Tratamento da Classe II esquelética, mordida aberta anterior, com aparelho de Thurow. Revista da SOB / Sociedade Brasileira de Ortodontia, Rio de Janeiro, v. 2, n. 4, p.115-118, 1993.

9. ORTON, H. E.; SLATTERY, DA.; ORTON, S. The treatment of severe 'gummy' Class II division 1 malocclusion with the maxillary intrusion splint. Eur J Orthod, London, v.14, p. 216-223, 1992.

10. ORTON, H. E.; BATTAGEL, J. M.; FERGUSON, R.; FERMAN, A. M. Distal movement of buccal segments with "en masse" removable appliance - Its value in treating patients with mild Class II, Division 1 malocclusions. Part I: Clinical techniques (how to do it). Am J Orthod Dentofacial Orthop, St. Louis, v. 109, no. 3, p. 234-243, 1996.

11. ORTON, H. E.; BATTAGEL, J. M.; FERGUSON, R.; FERMAN, A. M. Distal movement of buccal segments with "en masse" removable appliance - Its value in treating patients with mild Class II, Division 1 malocclusions. Part II: the model measuring system and results. Am J Orthod Dentofacial Orthop, St. Louis, v. 109, no. 4, p. 379-385, 1996.

12. SECKIN, Ö.; SÜRÜCÜ, R. Treatment of Class II, division 1, cases with a maxillary traction splint. Quintessence Int, Berlin, v. 3, p.17-23, 1990.

13. THUROW, R. C. Craniomaxillary orthopedic correction with en masse dental control. Am J Orthod St Louis, v. 68, no 6, p. 601-624, 1975.

14. ÜNER, O.; YÜCEL-EROGLU, E. Effects of a modified maxillary orthopaedic splint: a cephalometric evaluation. Eur J Orthod, London, v.18, p. 269-286, 1996.

\author{
Endereço para correspondência \\ Daltro Ritter \\ Rua Nereu Ramos 69, Sala 512 \\ Florianópolis - SC - Cep: 88015-010 \\ E-mail: daltroritter@hotmail.com
}

